\title{
MODELING AND CALCULATION OF WELDED STIFFENERS IN FABRICATION OF LARGE-SIZED ITEMS FOR METALLURGICAL PRODUCTION
}

\author{
A.V. LOZA, V.V. CHIGAREV and A.N. SERENKO \\ Priazovsky State Technical University \\ 7 Respublika Lane, 87500, Mariupol, Ukraine. E-mail: loza_a_v@pstu.edu
}

\begin{abstract}
The main causes for formation of cracks and local sagging in cast large-sized case structures used in metallurgy are considered. Modeling of stress-strain state of the most loaded zones of slag carrier bowls in operation was performed. It is shown that to reduce residual deformations of cast bowls, it is necessary to apply additional stiffeners in the form of welded-on ribs. Calculation was used to determine the required parameters of stiffeners and welds. 7 Ref., 8 Figures.
\end{abstract}

\section{Ke y w o r d s : cast items, thermal loads, slag carrier bowl, deformation, stiffeners}

Case parts, metallurgical vessels and other complex-shaped components, operating at high temperatures, are used in metallurgy and mechanical engineering. Casting is usually applied for their manufacture as the least expensive method of production of complex-shaped parts. At the same time, cast steel products are characterized by presence of diverse, both internal and external defects, which weaken the structure strength, are sources of crack initiation, and may lead to a change of the specified profile and early failure of the equipment or may cause an emergency. Therefore, solving the problem of ensuring the reliability of cast items in operation is an important engineering task.

Application of additional stiffeners is a rational and technologically convenient technique of structure reinforcement. In large-sized items, however, for instance metallurgical slag carrier bowls (Figure 1), stiffeners produced by casting have inherent metallurgical defects [1], that is why they do not ensure an

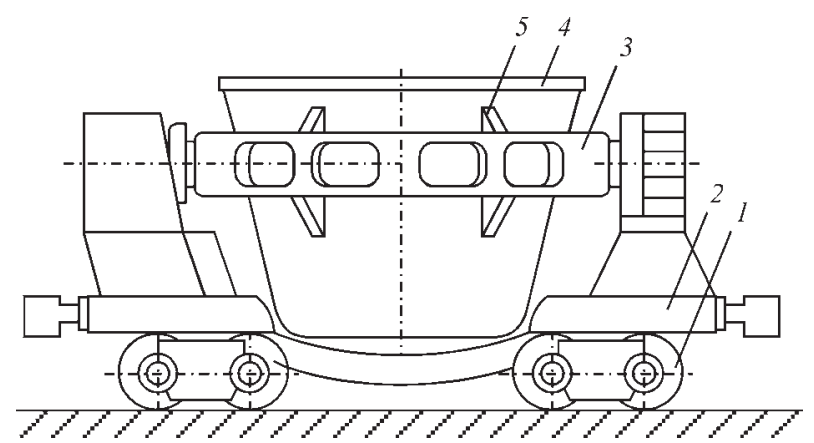

Figure 1. Slag carrier diagram: 1 - undercarriage; 2 - platform; 3 - support ring for bowl; 4 - bowl; 5 - support elements on the bowl increase of the part strength, particularly under the conditions of complex loading.

In-service cracks in the bowls very often form along the cast stiffeners, or defects associated with them, even in the case of local reinforcement of the structure (Figure 2).

Strengthening of large-sized items, due to application of expensive alloying elements, is not always rational. As it is difficult to predict the site of metallurgical defect location in cast components in practice, and impossible to prevent crack propagation, it is technologically convenient to enhance the item strength using additional structural elements, fastened by welding. They have the following main advantages:

- possibility to apply material with required properties;

- uniformity of properties over the section, that is achieved by application of rolled billets instead of cast ones.

Designing such elements to reinforce the structure, envisages fulfillment of two stages:

- designing the reinforcing element (stiffener);

- calculation of a welded joint for its fastening.

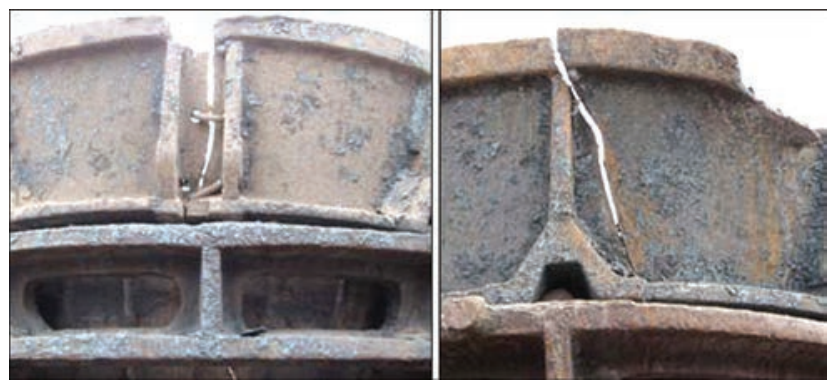

Figure 2. Cracks in slag bowls in the area of cast stiffeners 




Figure 3. Formation of residual deformations of the bowl in the form of «tightening»

Practical operation of equipment shows that early failure of a part or development of residual deformations are associated with its use under critical conditions [2]. Therefore, design of a strengthening element, for instance, additional stiffener in case of the need to increase the structure rigidity, should be performed allowing for maximum loads, which can be applied to this equipment in non-standard modes. For instance, design temperature of bowl case outer wall is about $450{ }^{\circ} \mathrm{C}$. Under production conditions, however, such situations arise occasionally, when the slag carrier or a train of several slag carriers is forced to stay in one place for some time after loading (receiving molten slag of $1500-1600{ }^{\circ} \mathrm{C}$ ). In this case the slag bowl case is exposed to non-uniform heating of the walls up to temperatures higher than the design ones. In the vicinity of slag carrier support ring, the temperature of the bowl outer surface can be $620^{\circ} \mathrm{C}$. Mechanical properties of case material (usually, carbon steels) are temperature-dependent characteristics [3]. High-temperature operation of steel items leads to weakening of grain boundaries [4]. At heating of the case above $580-600{ }^{\circ} \mathrm{C}$, carbon steel yield point

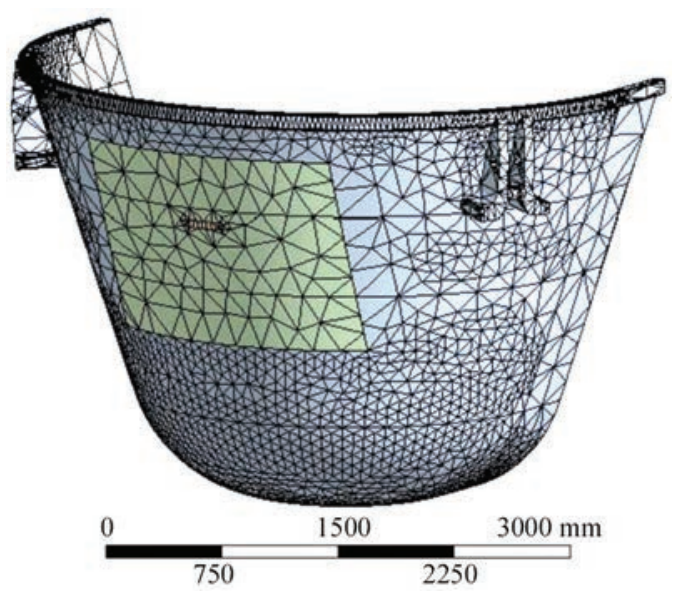

Figure 4. FEM-modeling of bowl case decreases considerably, that leads to development of residual deformations in the form of wall bending-in, its thinning and crack initiation.

In slag carrier operation, 3 to 4 months after the start of operating period, plastic deformations arise in the region of steering knuckle (so-called «tightening»), which develop with time (Figure 3).

Placing additional stiffeners is effective, if their welding point is maximum close to the zone of the highest deformations of bowl case. To assess development of the above defects, it is necessary to perform calculation of stress-strain state (SSS) of the slag carrier most loaded component - the bowl. Such a calculation can be performed, for instance, using the procedure of [5]. This allows determination of item regions, which need reinforcement to be able to operate at considerable thermal overloads.

Bowl state under the impact of process loads was modeled. Principles of frame design in a Cartesian system of coordinates were applied to develop a 3D geometrical model of slag carrier bowl (Figure 4).

Temperature distribution in the case of slag carrier bowl was determined when solving the thermal problem. During computation, the program compares the heat balance with the convergence criterion, specified in the problem. Checking results convergence was based on analysis of temperatures and heat flows. Results of solving the thermal problem are used to determine the strains.

During modeling it turned out that case deformations arise from non-equilibrium heating of wall material. Temperature of case outer surface is $420-620^{\circ} \mathrm{C}$, that of inner working surface is $800-900^{\circ} \mathrm{C}$. Thermal loads lead to bowl wall bending. Change of bowl case profile occurs in a certain zone of support ring steering knuckles that is critical in terms of preservation of the bowl working condition.

Calculation of temperature fields and arising deformations for the bowl with other (greater) case thicknesses show that increasing the bowl wall thickness at the same boundary conditions of the problem has virtually little impact on the values of wall bending-in. Therefore, improvement of bowl design by increasing its thickness is an unpromising and unprofitable direction.

It is established that in the case of local overheating of the wall above $600{ }^{\circ} \mathrm{C}$, case bending-in in this zone is inevitable (Figure 5) for bowls of currently available designs.

Cast stiffeners do not have the required ductility margin and cannot prevent residual deformations of the wall, and at cyclic variation of temperature they develop cracks, particularly in the presence of internal casting defects. Contrarily, stiffeners from rolled 


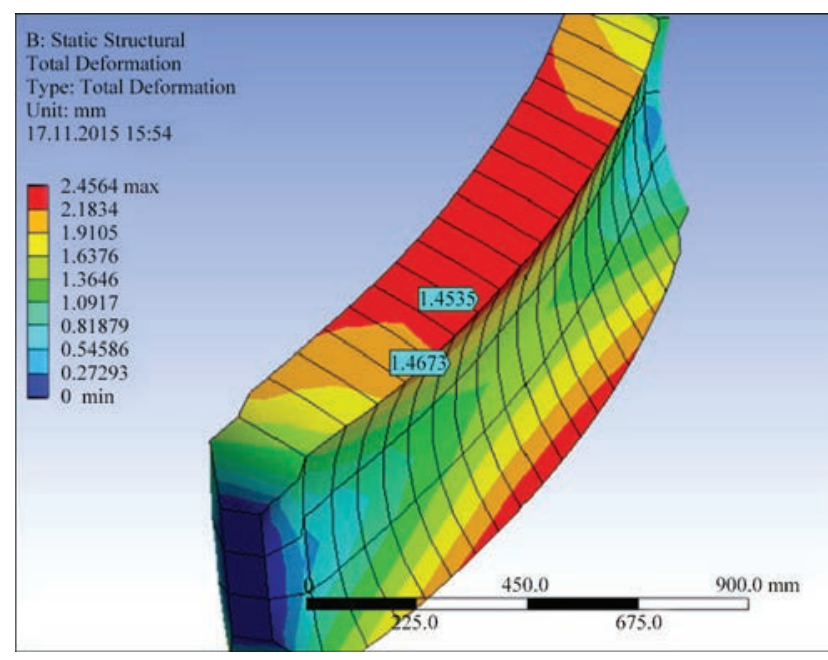

Figure 5. Modeling of behaviour of a section of slag carrier bowl wall in overheating zone in the area of support ring

metal with uniform properties, fastened by welding, have the required mechanical characteristics and are capable of solving the problem of ensuring the case rigidity at cyclic thermal and mechanical loads. The properties of such stiffeners can be variably assessed at the design stage, specifying in the calculation model the characteristics of the material of blanks from different steel grades.

To solve the problem of reinforcement of slag carrier bowl structure by welding stiffeners of required geometrical dimensions to it in specified zones, balancing load $F_{\text {bal }}$ was additionally determined first (Figure 6).

The problem is solved by finite element method (FEM). Here, balancing force $F_{\text {bal }}$ (estimated force) is determined, which can reduce the considered plate deformations to zero (or infinitely small values), i.e. to initial form of slag carrier bowl profile. Solving such a problem for the case of currently used bowl designs, yielded the value of design force $P_{1}=150 \mathrm{kN}$. Maximum force will be applied to welded stiffener in the horizontal plane at the level of stiffener lower edge.

Derived design force allowed determination of optimum shape and dimensions of additional reinforcing elements — stiffeners - with minimum material consumption. Several variants of geometrical dimensions were considered at analysis of stiffener design. Values of stiffener thickness of 10,16, 20, 30 and $40 \mathrm{~mm}$ were assigned. Stiffener loading was modeled in the case of application of balancing force along the horizontal (Figure 7, a). Stiffeners were separately tested for bending strength for the case of bowl resting on one stiffener (Figure 7, $b$ ) at load $P_{2}=120 \mathrm{kN}$ (half of bowl weight).

Modeling was performed with application of frame design technique. A section of bowl wall with greatest deformations was considered to solve the
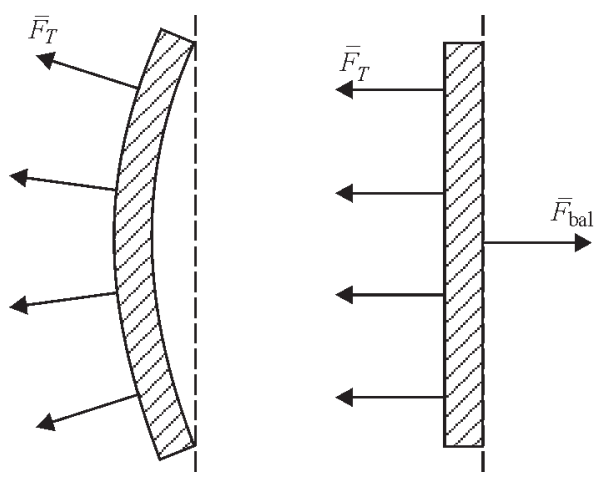

Figure 6. Diagram of load application to determine balancing force $F_{\text {bal }}$, leading to wall bending because of non-uniform heating system of equations. Stiffener fastening to the case by welding was modeled in the form of rigid connection along the line of stiffener and bowl contact. Analysis of SSS of several design variants allowed selecting such a variant, which has minimum values of stresses and possible strains under specified loads and boundary fastening conditions. Analysis showed that stiffener of $30 \mathrm{~mm}$ thickness corresponds to the strength condition.

Calculation of welds fastening the stiffener to slag carrier bowl (Figure 8) was performed for the case of application of fillet welds of tee-joints of T1 type by the procedure accepted in engineering practice [6, 7].

When selecting the scheme of force impact on welds of both the first and the second variant acc. to Figure 7 (Figure 8, $a, b$ ), it is necessary to take into account the fact that forces $P_{1}$ and $P_{2}$ are located at a distance from the welds, so that their stressed state will be determined by simultaneous action of the torque and the force directed at an angle to weld longitudinal axis. If we reduce all the forces to center $C$ and decompose $P_{1}$ and $P_{2}$ into normal $N$ and tangential $Q$ components (Figure $8, c, d$ ), we will obtain a plane system of forces applied to the weld:
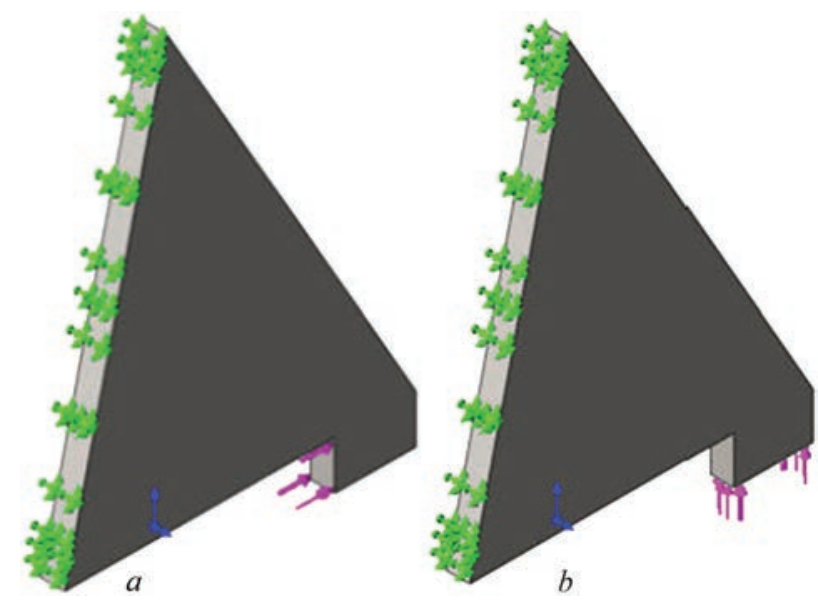

Figure 7. Scheme of calculation of bowl stiffener for first $(a)$ and second $(b)$ loading variants 

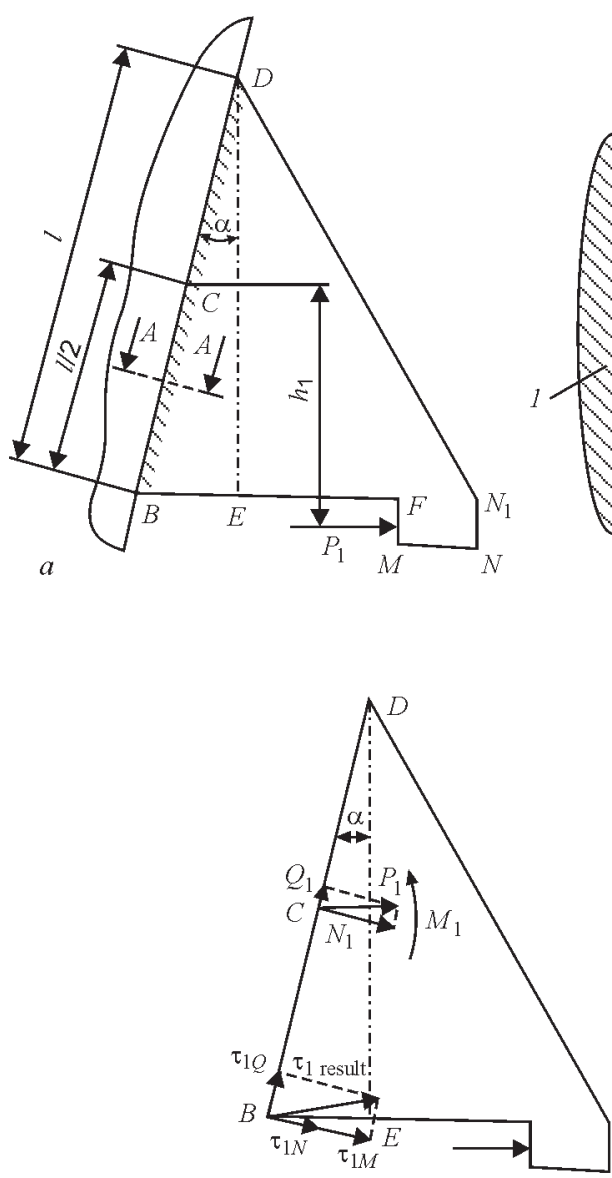

$c$
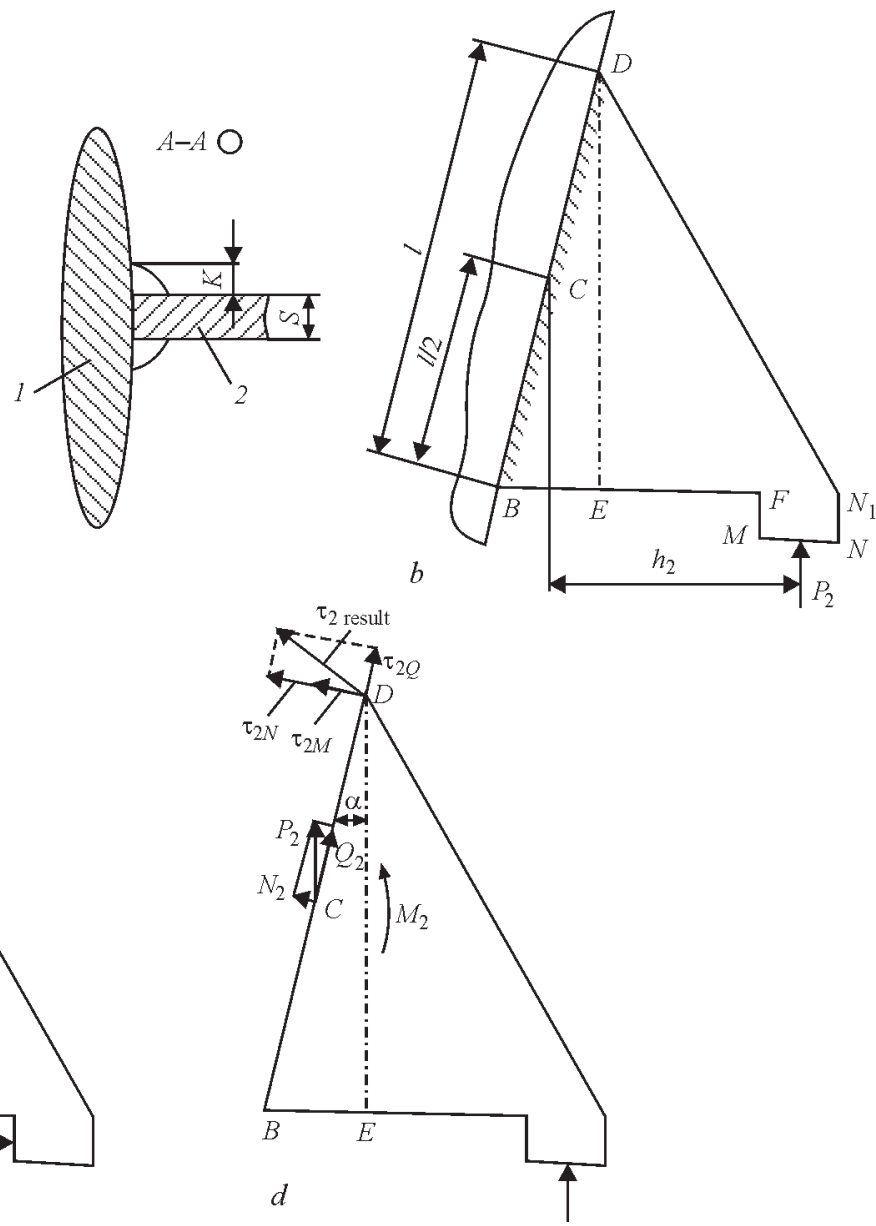

d

Figure 8. Scheme of welding stiffener to bowl and force impact on it: 1 - bowl wall; 2 - stiffener

$$
\begin{array}{ll}
M_{1}=P_{1} h_{1}, & M_{2}=P_{2} h_{2}, \\
N_{1}=P_{1} \cos \alpha, & N_{2}=P_{2} \sin \alpha, \\
Q_{1}=P_{1} \sin \alpha, & Q_{2}=P_{2} \cos \alpha,
\end{array}
$$

where $h_{1}, h_{2}$ are the arms of forces $P_{1}$ and $P_{2}$, respectively; $\alpha=\arcsin (B E / B D)$ is the angle of weld deflection from the vertical.

Highest stresses from the torque arise at weld ends (points $B$ and $D$ in Figure 8) and are given by the following formulas:

$$
\tau_{1 M}=\frac{M_{1}}{W_{1}}=\frac{P_{1} h_{1}}{W_{1}} ; \quad \tau_{2 M}=\frac{M_{2}}{W_{2}}=\frac{P_{2} h_{2}}{W_{2}},
$$

where $W_{1}, W_{2}$ are the moments of resistance of calculated sections of fillet welds for the first and second loading variants.

Shear area of fillet welds is determined by calculated thickness of fillet weld $h_{\text {calc }}$ and its length. Calculated thickness of the weld depends on the method of making the weld, its kind and is connected with weld leg by the following dependence:

$$
h_{\text {calc }}=\beta K \text {, }
$$

where $\beta$ is the coefficient for determination of calculated thickness of fillet weld, taken to be equal to: 1.1 for single-pass automatic welding; 0.9 for two- and three-pass automatic welding; and 0.7 for manual welding.

Moments of resistance of calculated sections of welds in T-joint are given by the following dependencies:

$$
W_{1}=\frac{2 h_{1 \mathrm{calc}} l^{2}}{6}=\frac{\beta K_{1} l^{2}}{3} ; \quad W_{2}=\frac{2 h_{2 \mathrm{calc}} l^{2}}{6}=\frac{\beta K_{2} l^{2}}{3},
$$

where $K_{1}, K_{2}$ are the weld legs calculated at the first and second loading variants; $l$ is the length of welds in the joint.

Taking into account all the above formulas, it is possible to find the components of tangential stresses from all the force factors for point $B$ and $D$ (Figure 8, $c, d)$ :

$$
\begin{gathered}
\tau_{1 M}=\frac{3 P_{1} h_{1}}{\beta K_{1} l^{2}}, \quad \tau_{1 N}=\frac{P_{1} \cos \alpha}{2 \beta K_{1} l}, \\
\tau_{1 Q}=\frac{P_{1} \sin \alpha}{2 \beta K_{1} l}, \quad \tau_{2 M}=\frac{3 P_{2} h_{2}}{\beta K_{2} l^{2}}, \\
\tau_{2 N}=\frac{P_{2} \sin \alpha}{2 \beta K_{2} l}, \quad \tau_{2 Q}=\frac{P_{2} \cos \alpha}{2 \beta K_{2} l} .
\end{gathered}
$$

Then, the resulting tangential stresses in the most stressed points of the weld, i.e. $B$ for the first loading 
variant and $D$ for the second loading variant, will be determined from the following formulas:

$$
\begin{gathered}
\tau_{\text {1result }}=\sqrt{\left(\tau_{1 M}+\tau_{1 N}\right)^{2}+\tau_{1 Q}^{2}}= \\
=\frac{P_{1}}{\beta K_{1} l} \sqrt{\left(\frac{3 h_{1}}{l}+\frac{\cos \alpha}{2}\right)^{2}+\frac{\sin ^{2} \alpha}{4}} ; \\
\tau_{2 \text { result }}=\sqrt{\left(\tau_{2 M}+\tau_{2 N}\right)^{2}+\tau_{2 Q}^{2}}= \\
=\frac{P_{2}}{\beta K_{2} l} \sqrt{\left(\frac{3 h_{2}}{l}+\frac{\sin \alpha}{2}\right)^{2}+\frac{\cos ^{2} \alpha}{4} .}
\end{gathered}
$$

Considering that the resultant stress vector is not normal to weld longitudinal axis, its calculated thickness and, therefore, weld leg should be increased $\gamma$ times, i.e. $\gamma_{1} K_{1}$ and $\gamma_{2} K_{2}$ instead of $K_{1}$ and $K_{2}$ should be assumed in (6) and (7), where

$$
\begin{aligned}
& \gamma_{1}=\frac{\sqrt{\left(\tau_{1 M}+\tau_{1 N}\right)^{2}+\tau_{1 Q}^{2}}}{\left(\tau_{1 M}+\tau_{1 N}\right)}= \\
& =\frac{\sqrt{\left(\frac{3 h_{1}}{l}+\frac{\cos \alpha}{2}\right)^{2}+\frac{\sin ^{2} \alpha}{4}}}{\frac{3 h_{1}}{l}+\frac{\cos \alpha}{2}} ; \\
& \gamma_{2}=\frac{\sqrt{\left(\tau_{2 M}+\tau_{2 N}\right)^{2}+\tau_{2 Q}^{2}}}{\tau_{2 M}+\tau_{2 N}}= \\
& =\frac{\sqrt{\left(\frac{3 h_{2}}{l}+\frac{\sin \alpha}{2}\right)^{2}+\frac{\cos ^{2} \alpha}{4}}}{\frac{3 h_{2}}{l}+\frac{\sin \alpha}{2}} .
\end{aligned}
$$

Substituting (8) and (9) into (6) and (7), after transformations we can write the strength condition for both the loading variants in the following form:

$$
\begin{aligned}
& \tau_{\text {1result }}=\frac{P_{1}}{\beta K_{1} l} \sqrt{\left(\frac{3 h_{1}}{l}+\frac{\cos \alpha}{2}\right)^{2}} \leq\left[\tau^{\prime}\right] ; \\
& \tau_{\text {2result }}=\frac{P_{2}}{\beta K_{2} l} \sqrt{\left(\frac{3 h_{2}}{l}+\frac{\sin \alpha}{2}\right)^{2}} \leq\left[\tau^{\prime}\right],
\end{aligned}
$$

where $\left[\tau^{\prime}\right]$ are the admissible stresses in the weld in shear.
We will obtain from expressions (10) and (11) the following formulas for weld leg determination:

$$
\begin{aligned}
& K_{1} \geq \frac{P_{1}}{\beta\left[\tau^{\prime}\right] l} \sqrt{\left(\frac{3 h_{1}}{l}+\frac{\cos \alpha}{2}\right)^{2}} ; \\
& K_{2} \geq \frac{P_{2}}{\beta\left[\tau^{\prime}\right] l} \sqrt{\left(\frac{3 h_{2}}{l}+\frac{\sin \alpha}{2}\right)^{2}} .
\end{aligned}
$$

Proceeding from design considerations, stiffener dimensions (in $\mathrm{mm}$ ) are taken to be as follows (see Figure 8): $B D=550, D E=534, B E=125, B F=330$, $M N=100, F M=60, h_{1}=0.5(D E \pm F M), h_{2}=B F-$ $0.5 B E+0.5 M N$. Weld length is $l=B D$. Stiffener material is St3 steel. Welds are made with E42 electrodes for manual arc welding; $\beta=0.7 ;\left[\tau^{\prime}\right]=0.6[\sigma]=$ $=96 \mathrm{MPa}$.

Substituting into formulas (12) and (13) the required magnitudes of values included into them, we obtain the following dimensions of weld leg: for the first loading variant (see Figure 7,a) $K_{1} \geq 8.55 \mathrm{~mm}$; for the second loading variant (see Figure $7, b$ ) $K_{2} \geq 5.62 \mathrm{~mm}$.

At simultaneous impact of both the forces, that is theoretically possible at hot bowl returning to initial position after tilting at the moment of slag unloading, $K \geq 14.17 \mathrm{~mm}$.

Allowing for possible scatter of real weld leg dimensions, technological dimension $K=15 \mathrm{~mm}$ is recommended.

Industrial trials of bowls with welded stiffeners confirmed the rationality of the proposed method for lowering thermal deformations of large-sized cast items.

1. Zhegur, A.A., Repyakh, S.I. (2010) On supply of T-shaped thermal centers in investment casting. Lit. Proizvodstvo, 12, 15-19.

2. Artyukh, V.G. (2008) Loads and overloads in metallurgical machines: Monography. Mariupol: PGTU.

3. (1989) Reference book on grades of steels and alloys. Ed. by V.G. Sorokin. Moscow: Mashinostroenie.

4. Goritsky, V.M. (1987) On causes of crack formation in bodies of oxygen-blow vessels. Metallurg. i Gornor. Promyshlennost, 1, 57-60.

5. Loza, A.V., Chigarev, V.V., Rassokhnin, D.A. et al. (2015) Study of deformation in final slabs during continuous steel casting. Izvestiya Vuzov. Chyorn. Metallurgiya, 58(3), 197202.

6. Nikolaev, G.A., Vinokurov, V.A. (1990) Welded structures. Calculation and design: Manual. Moscow: Vysshaya Shkola.

7. Serenko, A.N., Krumboldt, M.N., Bagryansky, K.V. (1977) Calculation of welded joints and structures. Examples and problems. Kiev: Vyshcha Shkola. 